\title{
Early diagnosis of renal dysfunction in hypertensive patients with type 2 diabetes mellitus
}

\author{
Oleksandr M Bilovol, Iryna I. Kniazkova, Oleksandr M. Kirienko, Vladyslav. I. Korniichuk, \\ Denis A. Kirienko, Lilya P. Abramova, Alexander E Berezin*
}

Received: 18 August 2020 / Received in revised form: 10 December 2020, Accepted: 12 December 2020, Published online: 19 December 2020 (C) Biochemical Technology Society 2014-2020

(C) Sevas Educational Society 2008

\begin{abstract}
Objective: to investigate intra-renal hemodynamic parameters and their relationship with the plasma level of neutrophil gelatinaseassociated lipocalin (NGAL) and cystatin $\mathrm{C}$ in patients with hypertension and type 2 diabetes mellitus. Materials and methods: 103 patients ( 45 men and 58 women) aged from 35 to 64 years (average age $55.5 \pm 3.4$ years) were examined, of which 55 ( 24 men, 31 women, average age $55.9 \pm 3.5$ years) were with stage II hypertension (AH) and type 2 diabetes mellitus (T2DM) of moderate severity, subcompensated (group 1) and 48 (21 men, 27 women, average age 55,4 $\pm 3,3$ years) were with stage II AH without concomitant T2DM (group 2). The control group included 25 apparently healthy individuals (average age $55.1 \pm 3.1$ years). All patients underwent routine clinical examination, determination of parameters of carbohydrate metabolism and lipid profile; insulin resistance was assessed by the HOMA-IR index. Using kits for enzyme immunoassay the concentration of renal dysfunction markers, cystatin $\mathrm{C}$ and neutrophilic gelatinase-associated lipocalin (NGAL), was measured in blood serum. Renal hemodynamic states were analyzed using B-type ultrasound and Doppler of the renal arteries. Results: The serum level of cystatin $\mathrm{C}$ when compared with the control group significantly increased in both groups of examined patients, reaching the highest values in comorbidity AH and T2DM-. In patients with AH and T2DM compared with patients without T2DM, there were higher levels of NGAL in serum. In patients with AH in combination with T2DM, a significant decrease in the end-diastolic velocity of arterial blood flow (Ved) was observed in comparison with patients with $\mathrm{AH}$ without T2DM. A substantial increase in the resistance index (RI) was revealed in patients of both groups in comparison with the control group. In addition, RI significantly increased in the group of patients with $\mathrm{AH}$ and T2DM compared with the group of patients with AH without T2DM. Conclusion: Among hypertensive patients at the stage of normoalbuminuria with preserved GFR NGAL and cystatin $\mathrm{C}$ were higher in T2DM
\end{abstract}

Oleksandr M Bilovol, Iryna I. Kniazkova, Oleksandr M. Kirienko, Vladyslav.I. Korniichuk, Denis A. Kirienko, Lilya P. Abramova

Kharkiv National Medical University, Kharkiv, Ukraine.

\section{Alexander E Berezin*}

State Medical University of Zaporozhye, Ukraine.

*E-mail: aeberezin @_gmail.com compared with non-T2DM. This corresponded to an increase in the resistance of the renal vascular bed in patients of both groups more pronounced in comorbidity with $\mathrm{AH}$ and T2DM.

Keywords: hypertension; chronic kidney disease; type 2 diabetes mellitus; cystatin C; neutrophil associated gelatinase lipocalin.

\section{Introduction}

Currently, diabetes mellitus (DM) is one of the most pressing problems facing medical science and healthcare worldwide (Buse et al., 2020; Ahmed et al., 2020; Bahshwan et al., 2019). The severity of type $2 \mathrm{DM}$ (T2DM) is due to generalized damage to the vascular system with the development of multiple microvascular (nephropathy, retinopathy) and macrovascular complications (coronary heart disease, peripheral atherosclerosis) (Kaur et al., 2018; Leung et al., 2016). The most dangerous consequences of diabetes are its vascular complications, among which diabetic nephropathy (DN) is a special problem, leading to the development of end-stage chronic renal failure and being one of the main causes of disability and mortality in T2DM patients (Khattab et al., 2020). Numerous data have been accumulated on the high prevalence of arterial hypertension (AH) in patients with T2DM (Yildiz et al., 2020). The comorbidity of hypertension and T2DM leads to an increased mutual influence on the course of diseases, the nature and severity of complications, causing remodeling of the vascular system of the kidneys, neurohormonal, inflammatory, and biochemical changes affecting the parenchyma and interstitium of the kidneys, aggravating apoptotic and fibrosing processes (Storz et al., 2018; Ruiz-Ortega et al., 2020).

The diagnosis of chronic kidney disease (CKD) in T2DM patients is based on the assessment of urinary albumin excretion microalbuminuria (Chen et al., 2019; Lessey et al., 2019). However, the non-specificity of this method in T2DM significantly reduces the discriminative value of the analysis for the diagnosis of diabetic kidney disease, since in T2DM the appearance of microalbuminuria is associated not only with CKD but also with multiple concomitant pathologies: hypertension, chronic heart failure, ischemic nephropathy, which can also lead to increased urinary albumin excretion.

Early diagnosis of acute kidney injury (AKI) is difficult due to the fact that up to $50 \%$ of kidney function can be lost even before creatinine rises (Shyam et al., 2017; Zayet et al., 2018). Timely and fast establishing diagnosis is vital to stratify a patient's risk and ensure appropriate treatment. In this regard, it is important to study 
the mechanisms of the development and progression of this pathology, to search for the earliest informative biomarkers reflecting early, clinically undetectable changes in the kidneys, and to carry out nephroprotection at the initial stages of CKD (Bidin et al., 2019). The most significant biomarkers of AKI include neutrophil gelatinase-associated lipocalin (NGAL) and cystatin C.

Most of the works devoted to the study of early biomarkers of renal damage in T2DM were carried out in animal models (Zhang et al., 2018). There is isolated research on the study of indicators of markers of preclinical diagnosis of diabetic kidney disease (Żyłka et al., 2015; Looker et al., 2018). Until now, early biomarkers of kidney damage in the comorbidity of T2DM and AH have not been evaluated, which suggests further study of the prognostic value of biomarkers of renal dysfunction in patients with initial manifestations of renal failure. The use of AKI biomarkers in combination with an imaging method for studying the kidneys (duplex scanning of the renal arteries) can make it possible to diagnose AKI with high accuracy at an early stage of its development. The objective of the study was to investigate intrarenal hemodynamics and their relationship with the plasma level of neutrophil gelatinase-associated lipocalin (NGAL) and cystatin $\mathrm{C}$ in patients with $\mathrm{AH}$ and type T2DM.

\section{Materials and Methods}

The study involved 103 patients (45 men and 58 women) aged 35 to 64 years (the average age $-55.5 \pm 3.4$ years), undergoing treatment in Government Institution "L.T.Malaya Therapy National Institute of the National Academy of Medical Sciences of Ukraine", which is the clinical base of the Department of Clinical Pharmacology and Internal Medicine of Kharkiv National Medical University.

The 1st group (group 1) consisted of 55 patients with a combined course of AH of II degree and T2DM (24 men, 31 women, average age $-55.9 \pm 3.5$ years). Group 2 (comparison group) included 48 patients with AH II degree ( 21 men, 27 women, average age 55.4 \pm 3.3 years). The control group included 25 healthy volunteers (10 men, 15 women, average age was $55.1 \pm 3.1$ years).

All the patients were examined in accordance with the recommendations of the European Society of Hypertension and the European Society of Cardiology (ESH/ESC, 2018), the American Diabetes Association (ADA) 2019, and the International Diabetes Federation (IDF) 2018.

All respondents signed informed consent to participate in the study.

The patient groups were comparable in terms of age, sex, duration of hypertension, office systolic (SBP), and diastolic blood pressure (DBP), heart rate (HR), body mass index (BMI). The enrollment in the study in patients with $\mathrm{AH}$ and T2DM the level of glycosylated hemoglobin (HbAlc) was $<8.0 \%$, achieved with diet and oral glucose-lowering drugs, in accordance with clinical recommendations (American Diabetes Association, 2019).

The criteria for excluding patients from the study were: inability or unwillingness of the patient to comply with the conditions of the study; type 1 diabetes mellitus; secondary hypertension; acute myocardial infarction or transient ischemic attack less than 12 months old; heart failure III-IY functional class (NYHA); HbA1c $>8.0 \%$; retinopathy III-IV degree; stage III diabetic nephropathy; diabetic foot syndrome; plasma potassium level of $<3.3$ or $>5.5$ $\mathrm{mmol} / \mathrm{L}$; anemia (hemoglobin less than $110 \mathrm{~g} / \mathrm{l}$ ); clinically significant diseases of the respiratory system, gastrointestinal tract, nervous system, kidney, and blood system in the acute stage, requiring drug treatment or significantly affecting the assessment of the studied parameters; malignant and autoimmune diseases.

The initial characteristics of the groups are shown in Tables 1 and 2.

Table 1: Basic demographic, clinical and anthropometric characteristics of patient study population

\begin{tabular}{|c|c|c|c|c|c|}
\hline \multirow[b]{2}{*}{ Variables } & \multirow[b]{2}{*}{$\begin{array}{l}\text { Healthy volunteers } \\
\qquad(\mathrm{n}=25)\end{array}$} & \multirow[b]{2}{*}{$\begin{array}{l}\text { Entire patient cohort } \\
\qquad(\mathrm{n}=103)\end{array}$} & \multicolumn{2}{|c|}{ Hypertensive patients $(\mathrm{n}=103)$} & \multirow[b]{2}{*}{ P-value } \\
\hline & & & $\begin{array}{c}\text { T2DM patients } \\
(\mathrm{n}=55)\end{array}$ & $\begin{array}{c}\text { Non-T2DM patients } \\
(\mathrm{n}=48)\end{array}$ & \\
\hline Age, year & $55.1(48-57)$ & $55.5(49-59)$ & $55.9(49-58)$ & $55.4(50-59)$ & NS \\
\hline Male, $\mathrm{n}(\%)$ & $10(40)$ & $45(43.7)$ & $24(43.6)$ & $21(43.7)$ & NS \\
\hline Dyslipidemia, n (\%) & - & 99 (96.1)\# & $53(96.4)$ & $46(95.8)$ & NS \\
\hline Smoking, n (\%) & $11(44)$ & $46(44.7)$ & $24(43.6)$ & $22(45.8)$ & NS \\
\hline Abdominal obesity, n (\%) & - & $24(23.3) \#$ & $13(23.6)$ & $11(22.9)$ & NS \\
\hline Microalbuminuria, n (\%) & - & - & - & - & NS \\
\hline LV hypertrophy, n (\%) & - & $65(63.1) \#$ & $39(70.9)$ & $26(54.2)$ & 0.001 \\
\hline BMI, $\mathrm{kg} / \mathrm{m}^{2}$ & $22.35 \pm 0.31$ & $24.61 \pm 1.53 \#$ & $24.93 \pm 1.38$ & $24.37 \pm 1.65$ & NS \\
\hline Waist circumference, $\mathrm{sm}$ & $78.5 \pm 2.51$ & $81.5 \pm 2.39$ & $82.3 \pm 2.43$ & $80.6 \pm 2.37$ & NS \\
\hline WHR, units & $0.78 \pm 0.03$ & $0.83 \pm 0.04$ & $0.84 \pm 0.04$ & $0.82 \pm 0.03$ & NS \\
\hline
\end{tabular}

Notes: data are given as mean \pm SD and median, \# - significant difference between healthy volunteers and entire patient' cohort. 
Table 2: Hemodynamics in patient study population

\begin{tabular}{|c|c|c|c|c|c|}
\hline \multirow[b]{2}{*}{ Variables } & \multirow{2}{*}{$\begin{array}{l}\text { Healthy volunteers } \\
\qquad(\mathrm{n}=25)\end{array}$} & \multirow{2}{*}{$\begin{array}{l}\text { Entire patient } \\
\text { cohort }(n=103)\end{array}$} & \multicolumn{2}{|c|}{ Hypertensive patients $(\mathrm{n}=103)$} & \multirow{2}{*}{$\begin{array}{c}\mathrm{P} \\
\text { value }\end{array}$} \\
\hline & & & $\begin{array}{l}\text { T2DM patients } \\
\qquad(\mathrm{n}=55)\end{array}$ & $\begin{array}{l}\text { Non-T2DM patients } \\
\qquad(\mathrm{n}=48)\end{array}$ & \\
\hline Office SBP, mm Hg & $122 \pm 3.5$ & $127 \pm 3.5$ & $128 \pm 3.4$ & $126 \pm 3.6$ & NS \\
\hline Office DBP, mm Hg & $74 \pm 2.5$ & $81 \pm 2.8$ & $82 \pm 3.3$ & $80 \pm 2.5$ & NS \\
\hline LVEF, \% & $64 \pm 4$ & $61 \pm 3 \#$ & $60 \pm 3$ & $61 \pm 3$ & NS \\
\hline LVMMI, g/m2 & $82.5 \pm 0.8$ & $133.1 \pm 1.2 \#$ & $138.7 \pm 1.1$ & $127.3 \pm 1,2$ & 0.05 \\
\hline GFR, $\mathrm{mL} / \mathrm{min} / 1.73 \mathrm{~m} 2$ & $103 \pm 2.51$ & $91 \pm 2.55$ & $90 \pm 2.63$ & $92 \pm 2.41$ & NS \\
\hline HOMA-IR & $1.68 \pm 0.59$ & $4.52 \pm 0.55 \#$ & $4.65 \pm 0.57$ & $4.43 \pm 0.53$ & NS \\
\hline Fasting glucose, $\mathrm{mmol} / \mathrm{L}$ & $4.29 \pm 0.25$ & $5.91 \pm 0.51 \#$ & $6.15 \pm 0.56$ & $5.41 \pm 0.52$ & NS \\
\hline HbA1c, $\%$ & $4.31 \pm 0.23$ & $6.15 \pm 0.67 \#$ & $6.28 \pm 0.89$ & $5,97 \pm 0.53$ & NS \\
\hline $\mathrm{TC}, \mathrm{mmol} / \mathrm{L}$ & $5.0 \pm 0.09$ & $5.71 \pm 0.09 \#$ & $5.73 \pm 0.08$ & $5.69 \pm 0.09$ & 0.05 \\
\hline $\mathrm{HDL}-\mathrm{C}, \mathrm{mmol} / \mathrm{L}$ & $1.31 \pm 0.09$ & $1.08 \pm 0.07 \#$ & $1.09 \pm 0.06$ & $1.08 \pm 0.08$ & NS \\
\hline $\mathrm{LDL}-\mathrm{C}, \mathrm{mmol} / \mathrm{L}$ & $2.84 \pm 0.08$ & $3.65 \pm 0.07 \#$ & $3.75 \pm 0.09$ & $3.53 \pm 0.07$ & 0.045 \\
\hline $\mathrm{TG}, \mathrm{mmol} / \mathrm{L}$ & $1.7 \pm 0.03$ & $2.11 \pm 0.04 \#$ & $2.23 \pm 0.07$ & $2.09 \pm 0.07$ & NS \\
\hline
\end{tabular}

Notes: data are given as mean \pm SD and median, \# - significant difference between healthy volunteers and entire patient' cohort.

All patients underwent antihypertensive therapy (a combination of generally accepted drug classes: ACE inhibitors, calcium antagonists, beta-blockers (carvedilol) in medium therapeutic doses). Also, all patients received rosuvastatin at a dose of $20 \mathrm{mg}$ and acetylsalicylic acid at a daily dose of $75 \mathrm{mg}$. Metformin was combined with gliclazide to correct glycemia.

\section{Ethical declaration}

Informed consent was obtained from all the patients for participation in the study.

\section{General anthropometric, clinical, and physical examinations}

Conventional anthropometric measurements (height, weight, waist circumference, hip-to-waist ratio, body mass index [BMI]) were performed. Clinical history was recorded and specified. All patients underwent general clinical and physical examination, office blood pressure (BP) and heart rate (HR) measure, as well as ambulatory BP monitoring at baseline.

Chronic kidney disease (CKD) was defined according to the Clinical Practice Guideline for the Evaluation and Management of Chronic Kidney Disease (KGIGO 2012) (Stevens and Levin, 2012).

\section{Calculation of glomerular filtration rate}

The functional state of the kidneys was assessed by the glomerular filtration rate (GFR) using the CKD-EPI (Chronic Kidney Disease Epidemiology Collaboration) formula (Schwandt et al., 2017).

Renal artery B-mode ultrasound
Duplex scanning of the renal arteries was carried out in the area of the distal parts of the main trunk of the renal artery using a GE Medical Systems ultrasound scanner (Germany) with a convex probe with a frequency of $2.25-3 \mathrm{MHz}$ and a probe with a frequency of 1-7 MHz in the modes of color Doppler mapping and pulse wave. To determine the state of renal hemodynamics, the peak systolic $(\mathrm{Vps}, \mathrm{cm} / \mathrm{s})$ and end-diastolic velocities of arterial blood flow (Ved, $\mathrm{cm} / \mathrm{s}$ ) were assessed.

The resistance index was determined by the formula: RI $=(\mathrm{Vps}$ Ved)/Vps (Rinehardt et al., 2014).

Biomarker determination

Determination of fasting serum glucose (FSC) concentration, glycosylated hemoglobin (HbA1c) level in whole blood, insulin, lipid profile parameters; insulin resistance was assessed using the HOMA-IR index. Commercial kits of enzyme-linked immunosorbent assay were used to measure the serum concentration of markers of renal dysfunction - cystatin C (Bio Vendor, Czech Republic) and neutrophil gelatinase-associated lipocalin (NGAL, Hycult Biotech, Netherlands).

\section{Statistics}

The statistical application package Statistics for Windows 10 was used for statistical analysis. All data are presented in the form of the mean $(\mathrm{M})$, standard deviation $( \pm \mathrm{SD})$, median $(\mathrm{Me})$, and interquartile range (IQR). The hypothesis about the normality of the distribution of the studied indicators was tested using the Shapiro-Willis test. To compare the statistical characteristics in different groups used multiple comparisons by one-way dispersion analysis of variance Kruskal-Wallis (ANOVA) with pair-wise comparison by the Mann-Whitney U test. For the analysis of $2 \times 2$ 
conjugation tables when comparing categorized variables, a twosided exact Fisher criterion or Chi2 test was used. To analyze the direction and strength of the relationship between certain indicators, we used the method of correlation analysis with the calculation of Spearman's coefficients. Differences were considered significant at values of $\mathrm{p}<0.05$

\section{Results}

Table 3 shows the content of cystatin $\mathrm{C}$ and NGAL in the blood of patients with a combination of $\mathrm{AH}$ and $\mathrm{T} 2 \mathrm{DM}$, as well as in the group of patients with $\mathrm{AH}$ and the control group.

Table 3: Content of the studied biomarkers in patients with hypertension and T2DM

\begin{tabular}{|c|c|c|c|}
\hline \multirow{2}{*}{ Variables } & $\begin{array}{c}\text { Control group } \\
\text { Healthy volunteers } \\
(\mathrm{n}=25)\end{array}$ & $\begin{array}{c}\text { Base group } \\
\text { AH+ T2DM } \\
(\mathrm{n}=55)\end{array}$ & $\begin{array}{c}\text { Comparison group } \\
\text { AH } \\
(\mathrm{n}=48)\end{array}$ \\
\hline \multirow{2}{*}{ Cystatin $\mathrm{C}, \mathrm{mg} / \mathrm{mL}$} & $83 \pm 12$ & $265 \pm 371 * * *$ & $108 \pm 111 * * * 2 *$ \\
& $(71.63-95.43)$ & $(249,0-281.15)$ & $(92.25-124.38)$ \\
\hline \multirow{2}{*}{ NGAL, $\mathrm{ng} / \mathrm{mL}$} & $9,63 \pm 0,71$ & $21,25 \pm 0,331 * * *$ & $17,13 \pm 0,211 * * * 2 *$ \\
& $(5.83-13.43)$ & $(18.17-23.39)$ & $(15.03-19.21)$ \\
\hline
\end{tabular}

Notes: $1 *$ - reliability of differences with the control group $(\mathrm{p}<0.05) ; 1 * * *-(\mathrm{p}<0.001) ; 2 *$ - significance of differences between the base and comparison groups $(\mathrm{p}<0.05)$.

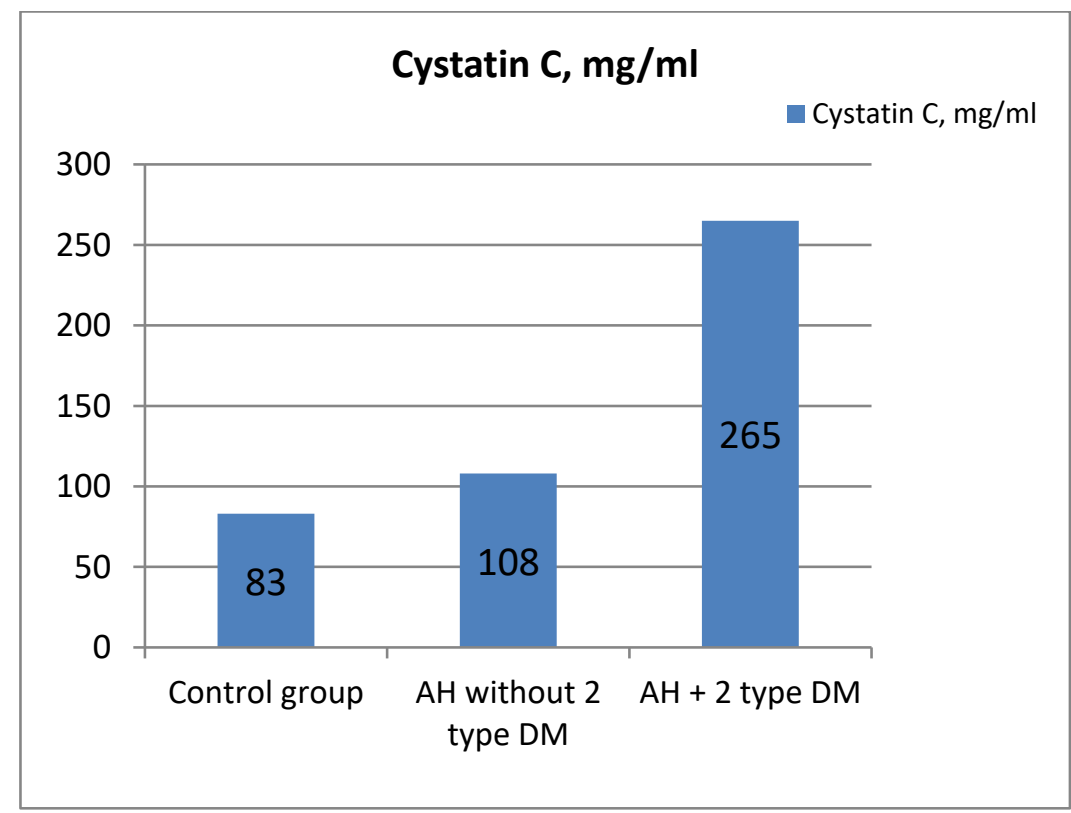

Figure 1: The content of cystatin $\mathrm{C}$ in the blood of patients with a combination of T2DM and hypertension, in the group of patients with hypertension, and in the control group $(\mathrm{mg} / \mathrm{ml})$.

It was noted that the level of cystatin $\mathrm{C}$ in the blood significantly increased both in the group of patients with type 2 diabetes and hypertension $(\mathrm{p}=0.04)$ and in the group of patients with hypertension ( $p=0.001$ ) compared with the control group. Figure 1 shows that an increase in the excretion of cystatin $\mathrm{C}$ is observed in patients with hypertension and increases with T2DM ( $\mathrm{p}=$ 0.001). The highest values of the level of cystatin $\mathrm{C}$ in the blood were determined in patients with T2DM, which exceeded the median values in the healthy control group by more than 3 times $(\mathrm{p}<0.001)$. 


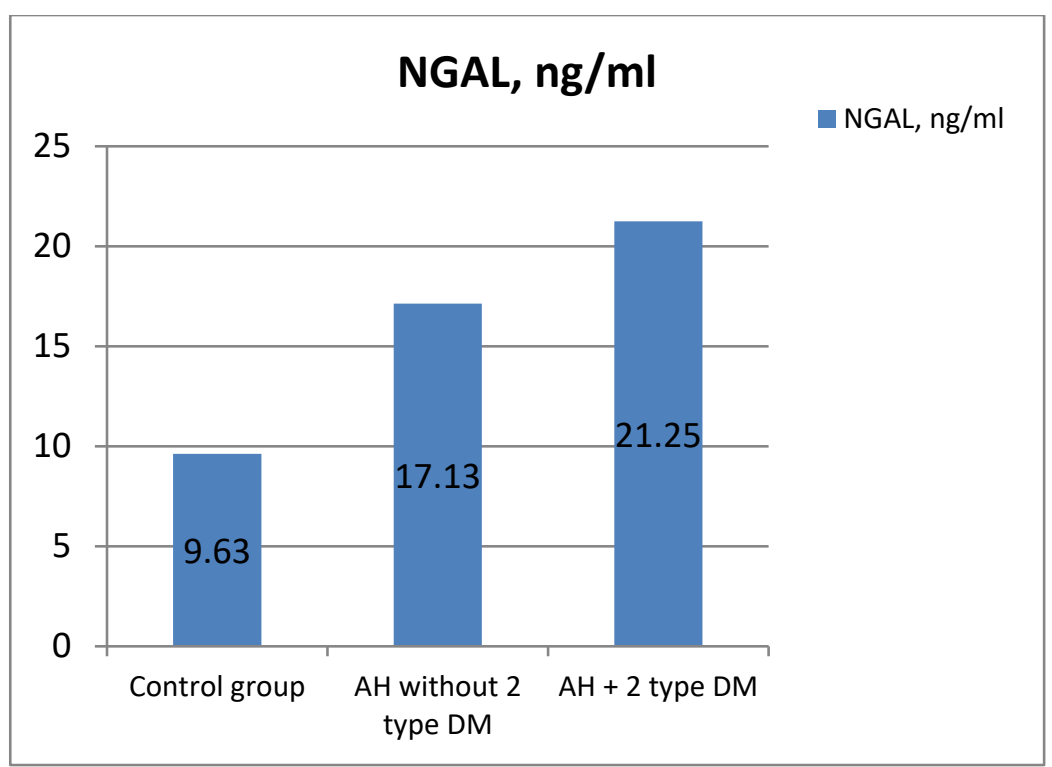

Figure 2: The content of NGAL in the blood of patients with T2DM, in the group of patients without T2DM, and in the control group (ng/ml).

As we can see in Figure 2, higher NGAL concentrations were found in hypertensive patients with T2DM compared with the group of relatively healthy individuals $(\mathrm{p}=0.001)$. NGAL levels differed significantly between patients in base and comparison groups $(\mathrm{p}=0.042)$. The concentrations of the studied biomarkers in patients of both groups did not differ significantly depending on gender, overweight, or obesity.

The data of duplex scanning of the renal arteries in the examined patients and healthy individuals are presented in Table 4.

Table 4: The data of renal hemodynamics in the examined groups

\begin{tabular}{|c|c|c|c|}
\hline \multirow[b]{2}{*}{ Variables } & \multirow{2}{*}{$\begin{array}{l}\text { Healthy } \\
\text { volunteers } \\
(\mathrm{n}=25)\end{array}$} & \multicolumn{2}{|c|}{ Hypertensive patients } \\
\hline & & $\begin{array}{l}\text { T2DM } \\
(\mathrm{n}=55)\end{array}$ & $\begin{array}{l}\text { Non-T2DM } \\
\quad(\mathrm{n}=48)\end{array}$ \\
\hline $\mathrm{Vps}, \mathrm{cm} / \mathrm{s}$ & $92.8 \pm 3,1$ & $105.0 \pm 4.1^{1 *}$ & $111.2 \pm 4.3^{1 *}$ \\
\hline Ved, $\mathrm{cm} / \mathrm{s}$ & $36.9 \pm 1,1$ & $27.1 \pm 1.3^{1 * * * 2 *}$ & $31.3 \pm 1.3^{1 * * *}$ \\
\hline RI, unit & $0.60 \pm 0,03$ & $0.79 \pm 0.031 * * * 2 *$ & $0.71 \pm 0.02^{1 * * *}$ \\
\hline
\end{tabular}

Notes: $1 *$ - reliability of differences with the control group $(\mathrm{p}=0.024) ; 1 * * *$. ( $p=0.001) ; 2 *$ - significance of differences between the base and comparison groups $(\mathrm{p}=0.042)$.

It was found that the peak systolic velocity of arterial blood flow (Vps) significantly increased in the group of patients with T2DM $(\mathrm{p}=0.02)$ and in the group of patients with AH without T2DM ( $p$ $=0.02$ ) compared with the control group. At the same time, there were no significant differences in peak systolic arterial blood flow (Vps) between the base and comparison groups.

Analysis of the end-diastolic velocity of arterial blood flow (Ved) showed a significant decrease in the measurements of patients with T2DM and in the group of patients without diabetes compared with the control group. There was a significant decrease in Ved in patients with T2DM compared with the group of patients without diabetes. This was consistent with a significant increase in the resistance index (RI) in patients of both groups in comparison with the control group. At the same time, RI significantly increased in the group of patients with T2DM compared with the group of patients without diabetes.

Thus, in patients with T2DM, a decrease in Ved and an increase in RI can serve as markers in the diagnosis of preclinical renal dysfunction.

Correlation analysis in patients with T2DM established a statistically significant correlation between NGAL level and serum creatinine concentration $(\mathrm{r}=0.35 ; \mathrm{p}=0,042)$. A statistically significant correlation was found between the level of cystatin $\mathrm{C}$ and the concentration of creatinine in the blood serum $(r=0.38 ; p$ $=0.044)$ and a negative correlation with the CKF EPI $(r=-0.31 ; p$ $=0.038$ ).

In hypertensive patients with T2DM, statistically significant positive correlations were found between RI and cystatin $\mathrm{C}$ level $(r=0.43 ; p=0.04)$, NGAL level in serum $(r=0.41 ; p=0.044)$, serum creatinine concentration $(r=0.36 ; p=0.01)$, as well as between Ved and GFR EPI $(r=0.31 ; p=0.01)$. In addition, significant negative correlations were noted between Ved and cystatin $\mathrm{C}$ level $(\mathrm{r}=-0.41 ; \mathrm{p}=0.001)$, NGAL level in serum $(\mathrm{r}=$ 0.40; $\mathrm{p}$ < 0.001), as well as between RI and CKF EPI ( $\mathrm{r}=-0.32 ; \mathrm{p}$ $=0,001)$.

\section{Discussion}

Due to the progressive nature of the course of CKD, the nonspecificity of the microalbuminuria parameter in renal pathology in patients with T2DM, and the limited effectiveness of therapy in the expressed stages of diabetic nephropathy, preclinical diagnosis of this complication, when changes in the kidneys are potentially reversible, is of special relevance. The search for laboratory 
markers that allow the detection of CKD in T2DM at preclinical stages is important for the timely identification of high-risk groups of CKD among patients with T2DM and effective preventive renal protection.

In recent years, more and more importance in the pathogenesis of diabetic nephropathy is given to tubulointerstitial disorders, which according to a number of studies precede the development of glomerular lesions (Alicic et al., 2017). It was noted that in T2DM renal pathology is revealed with a predominant lesion of the interstitium and renal tubules, and the rate of decrease in renal filtration is associated with the severity of tubulointerstitial fibrosis, characterized by a progressive accumulation of extracellular matrix proteins. Its development is the result of activation of powerful cytokine proinflammatory cascades initiated by heterogeneous damaging factors: immune, ischemic, toxic, metabolic, impaired renal hemodynamics, hypertension, as well as a functional overload of the kidneys. In addition, the development of endothelial dysfunction leads to hemodynamic disturbances in the kidney and disturbances in the structure of the interstitium (Zannad and Rossignol, 2018).

In our study, statistically significant differences in biomarkers reflecting tubulointerstitial dysfunction were established between the group of healthy individuals and groups of patients with T2DM with normoalbuminuria. Thus, one of the markers of a functioning tubular mass is NGAL produced by epithelial cells and neutrophils, which is produced in the renal tubules in response to structural damage (Shang and Wang, 2017).

In our study, an increase in the content of NGAL in the blood plasma of patients with $\mathrm{AH}$ and with T2DM with normoalbuminuria was noted that, apparently, is due to damage to the renal tubules, which is characteristic of most patients with renal failure. Long-term expression of the NGAL gene in renal tubular cells can directly participate in the progression of kidney damage and be involved in pathological signaling pathways that cause the development of tubulointerstitial fibrosis (Nickolas et al., 2012).

Another marker of both tubular and glomerular damage is cystatin $\mathrm{C}$, a protease inhibitor produced by all nuclear cells, which is freely filtered in the kidney glomeruli and is almost completely reabsorbed and catabolized in the cells of the proximal tubules (Ferguson et al., 2015). A number of studies have shown that in T2DM, the level of cystatin $\mathrm{C}$ is a more exact marker of renal function than the level of creatinine and can be used for early detection of a decrease in RFR (Bargnoux et al., 2019).

As a marker of RFR serum cystatin C significantly exceeds serum creatinine and creatinine clearance, as it is able to: 1) diagnose the earliest changes in RFR (hyperfiltration and early stages of hypofiltration); 2) quickly track the decrease in GFR with the development of ARF; 3) exactly assess renal function in pediatric and geriatric patients; 4) predict cardiovascular and other complications of renal dysfunction in patients with T2DM; 5) is sensitive to the detection of early renal dysfunction.
In our study in hypertensive patients with T2DM the serum creatinine concentration and the glomerular filtration rate (according to the CKD-EPI formula) in most cases corresponded to the reference values, which testified in favor of the low diagnostic significance of these parameters for assessing the functional state of the kidneys in this category of patients. On the other hand, an increase in the level of cystatin $\mathrm{C}$ in the blood serum in patients with $\mathrm{AH}$ was found, which was more pronounced in T2DM. This suggested that the minimal changes in renal function determined by cystatin $\mathrm{C}$, even at the stage of normoalbuminuria, are an early marker of decreased renal function in T2DM.

According to the data of duplex scanning of the renal arteries an increase in the peripheral resistance index - the RI was noted, which was more pronounced in patients with T2DM and witnessing a greater increase in intraglomerular pressure and a faster progression of renal failure.

In addition, the blood flow velocity into systole in the examined patients significantly increased in comparison with the group of healthy individuals and did not differ significantly in patients with T2DM and in patients without diabetes. The end-diastolic velocity of arterial blood flow (Ved) significantly decreased in T2DM ( $\mathrm{p}=$ $0.001)$ and in the group of patients with $\mathrm{AH}$ without diabetes $(\mathrm{p}=$ 0.001 ) compared with the control group. At the same time, Ved significantly decreased in T2DM when compared with patients without T2DM.

So, in patients of both groups, there was an increase in the resistance of the vascular bed of the kidneys, more pronounced in the comorbidity of AH and T2DM.

\section{Conclusions}

In patients with $\mathrm{T} 2 \mathrm{DM}$ at the stage of normoalbuminuria with preserved RFR, more significant changes in biomarkers of renal dysfunction were noted compared with patients without T2DM. Thus, a significantly greater increase in the content of NGAL and cystatin C was noted, which indicates violations of both the glomerular and tubulointerstitial renal apparatus already at the preclinical stage of CKD. The revealed abnormalities in the level of biomarkers correlated with the concentration of creatinine in the blood serum and CKF EPI.

\section{Authors' contribution}

OMB designed the study protocol and proposed the idea of investigation. OMB, IMK, MVB, DAK, and OA obtained findings, analyzed the data, and reviewed the manuscript. AEB analyzed the data and wrote, edited, and reviewed the manuscript. MVB and LPA performed data collection, analyzed the statistics data, and checked the accuracy of the data analysis. IMK, LPA, and OMK performed an ultrasound examination and analyzed the results of it. OMK and LPA collect blood samples and performed the measurement of circulating biomarker concentrations. All authors read and approved the final version of the manuscript. 


\section{Acknowledgments}

We thank staff person of Clinical Pharmacology and Internal Medicine department (Kharkiv National Medical University, Kharkiv, Ukraine) and Government Institution "L.T.Malaya Therapy National Institute NAMSU" (Kharkiv, Ukraine), as well as patients for participation in the study. We also thank a team of Foreign Languages Department (Kharkiv National Medical University, Kharkiv, Ukraine) for assistance in manuscript preparation.

\section{Competing interests}

Authors have no competing interests

Ethics approval and consent to participate

The study was approved by the local ethics committee of Kharkiv National Medical University, Kharkiv, Ukraine. All patients gave their voluntary consent to participate in the study

Funding

This study was not supported by the non-commercial organization and funds. Kharkiv National Medical University was the only source of financial support.

\section{References}

Ahmed, I. A. B., Alosaimi, M. E., Alkhathami, S. M., Alkhurayb, N. T., Alrasheed, M. S., Alanazi, Z. M., ... \& Alazwary, M. N. (2020). Knowledge, attitude, and practices towards diabetes mellitus among non-diabetes community members of Riyadh, Kingdom of Saudi Arabia. International Journal of Pharmaceutical Research \& Allied Sciences, 9(1), 41-51.

Alicic, R. Z., Rooney, M. T., \& Tuttle, K. R. (2017). Diabetic kidney disease: challenges, progress, and possibilities. Clinical Journal of the American Society of Nephrology, 12(12), 2032-2045. doi: 10.2215/CJN.11491116.

American Diabetes Association. (2019). 2. Classification and diagnosis of diabetes: standards of medical care in diabetes-2019. Diabetes care, 42(Supplement 1), S13S28. doi: 10.2337/dc19-S002.

Bahshwan, S. M., Rabah, S. O. A., \& Turkistani, A. M. (2019) A Comparative Study of The Effect of Crude and Nanoparticles Costus Speciosus On Testicular Damage Associated to Experimentally Induced Type 2 Diabetes. Pharmacophore, 10(1), 99-106

Bargnoux, A. S., Barguil, Y., Cavalier, E., \& Cristol, J. P. (2019, August). Estimation of glomerular filtration rate using cystatin C. In Annales de biologie clinique (Vol. 77, No. 4, pp. 375-380). doi: 10.1684/abc.2019.1456.

Bidin MZ, Shah AM, Stanslas J, \& Seong CLT. (2019). Blood and urine biomarkers in chronic kidney disease: An update. Clin Chim Acta. 495:239-250. doi: 10.1016/j.cca.2019.04.069. Epub 2019 Apr 19.
Buse, J. B., Wexler, D. J., Tsapas, A., Rossing, P., Mingrone, G., Mathieu, C., ... \& Davies, M. J. (2020). 2019 Update to: Management of Hyperglycemia in Type 2 Diabetes, 2018. A Consensus Report by the American Diabetes Association (ADA) and the European Association for the Study of Diabetes (EASD). Diabetes Care. 43(2):487-493. doi: 10.2337/dci19-0066. Erratum in: Diabetes Care. 2020;43(7):1670; DOI: 10.2337/dc20-er07.

Chen TK, Knicely DH, \& Grams ME. (2019). Chronic Kidney Disease Diagnosis and Management: A Review. JAMA. 322(13):1294-1304. doi: 10.1001/jama.2019.14745.

Ferguson, T. W., Komenda, P., \& Tangri, N. (2015). Cystatin C as a biomarker for estimating glomerular filtration rate. Current opinion in nephrology and hypertension, 24(3), 295-300. doi: 10.1097/MNH.0000000000000115.

Kaur R, Kaur M, \& Singh J. (2018). Endothelial dysfunction and platelet hyperactivity in type 2 diabetes mellitus: molecular insights and therapeutic strategies. Cardiovasc Diabetol. 17(1):121. doi: 10.1186/s12933-018-0763-3..

Khattab, H. A., Moselhy, S. S., \& Aljafri, A. O. (2020). Olive Leaves Extract Alleviate Diabetic Nephropathy in Diabetic Male Rats: Impact on Oxidative Stress and Protein Glycation. International Journal of Pharmaceutical Research \& Allied Sciences, 9(1), 130-141

Lessey G, Stavropoulos K, \& Papademetriou V. (2019). Mild to moderate chronic kidney disease and cardiovascular events in patients with type 2 diabetes mellitus. Vasc Health Risk Manag.15:365-373. doi: 10.2147/VHRM.S203925.

Leung WK, Gao L, Siu PM, \& Lai CW. (2016). Diabetic nephropathy and endothelial dysfunction: Current and future therapies, and emerging of vascular imaging for preclinical renal-kinetic study. Life Sci. 166:121-130. doi: 10.1016/j.lfs.2016.10.015.

Looker HC, Mauer M, \& Nelson RG. (2018). Role of Kidney Biopsies for Biomarker Discovery in Diabetic Kidney Disease. Adv Chronic Kidney Dis. 25(2):192-201. doi: 10.1053/j.ackd.2017.11.004.

Nickolas, T. L., Forster, C. S., Sise, M. E., Barasch, N., Solá-Del Valle, D., Viltard, M., ... \& Mattei, S. (2012). NGAL (Lcn2) monomer is associated with tubulointerstitial damage in chronic kidney disease. Kidney international, 82(6), 718722. doi: 10.1038/ki.2012.195.

Rinehardt, E. K., Zierler, R. E., \& Leverson, G. E. (2014). Duplex scanning has a limited role in the evaluation of patients with renal failure. Journal of vascular surgery, 60(6), 15931598. doi: 10.1016/j.jvs.2014.08.109. Epub 2014 Oct 12. PMID: 25312535.

Ruiz-Ortega M, Rayego-Mateos S, Lamas S, Ortiz A, Rodrigues\& Diez RR. (2020). Targeting the progression of chronic kidney disease. Nat Rev Nephrol. 16(5):269-288. doi: 10.1038/s41581-019-0248-y.

Schwandt, A., Denkinger, M., Fasching, P., Pfeifer, M., Wagner, C., Weiland, J., ... \& Holl, R. W. (2017). Comparison of MDRD, CKD-EPI, and Cockcroft-Gault equation in relation to measured glomerular filtration rate among a large cohort with diabetes. Journal of Diabetes and its 
Complications, 31(9), 1376-1383. doi: 10.1016/j.jdiacomp.2017.06.016.

Shang, W., \& Wang, Z. (2017). The update of NGAL in acute kidney injury. Current Protein and Peptide Science, 18(12), 1211-1217. doi: 10.2174/1389203717666160909125004.

Shyam R, Patel ML, Sachan R, Kumar S, \& Pushkar DK. (2017). Role of Urinary Neutrophil Gelatinase-associated Lipocalin as a Biomarker of Acute Kidney Injury in Patients with Circulatory Shock. Indian J Crit Care Med. 21(11):740745. doi: 10.4103/ijccm.IJCCM_315_17.

Stevens, P. E., \& Levin, A. (2012). Kidney disease: improving global outcomes chronic kidney disease guideline development work group M. Evaluation and management of chronic kidney disease: synopsis of the kidney disease: improving global outcomes, 825-30. doi: 10.7326/00034819-158-11-201306040-00007.

Storz, C., Hetterich, H., Lorbeer, R., Heber, S. D., Schafnitzel, A., Patscheider, H., ... \& Reiser, M. (2018). Myocardial tissue characterization by contrast-enhanced cardiac magnetic resonance imaging in subjects with prediabetes, diabetes, and normal controls with preserved ejection fraction from the general population. European Heart Journal-
Cardiovascular Imaging, 19(6), 701-708. doi: 10.1093/ehjci/jex190.

Yildiz M, Esenboğa K, \& Oktay AA. (2020). Hypertension and diabetes mellitus: highlights of a complex relationship. Curr Opin Cardiol. 35(4):397-404. doi: 10.1097/HCO.0000000000000748.

Zannad, F., \& Rossignol, P. (2018). Cardiorenal syndrome revisited. Circulation, 138(9), 929-944. doi: 10.1161/CIRCULATIONAHA.117.028814.

Zayet, G. K. (2018). Serum Hepatocytes growth factor in acute and chronic Kidney diseasepatients and its relation to disease activity. Journal of Advanced Pharmacy Education \& Research. 8(3), 74-80.

Zhang ZH, He JQ, Qin WW, Zhao YY, \& Tan NH. (2018). Biomarkers of obstructive nephropathy using a metabolomics approach in rat. Chem Biol Interact. 296:229239. doi: 10.1016/j.cbi.2018.10.004.

Żyłka A, Gala-Błądzińska A, Rybak K, Dumnicka P, Drożdż R, \& Kuśnierz-Cabala B. (2015). Role of new biomarkers for the diagnosis of nephropathy associated with diabetes type 2 . Folia Med Cracov. 55(4):21-33. 